\title{
Philosophiques
}

\section{Critique de la pensée historique}

\section{Les premiers historiens français du mouvement ouvrier}

\section{Guy Massicotte}

Volume 9, numéro 1, avril 1982

URI : https://id.erudit.org/iderudit/203182ar

DOI : https://doi.org/10.7202/203182ar

Aller au sommaire du numéro

Éditeur(s)

Société de philosophie du Québec

ISSN

0316-2923 (imprimé)

1492-1391 (numérique)

Découvrir la revue

\section{Citer cet article}

Massicotte, G. (1982). Critique de la pensée historique : les premiers historiens français du mouvement ouvrier. Philosophiques, 9(1), 3-39.

https://doi.org/10.7202/203182ar
Résumé de l'article

L'historien a pour fonction de reconstituer le passé de manière à conjuguer les exigences de rigueur méthodologique et de pertinence par rapport aux questions qui intéressent les hommes de son temps. Parmi les divers agencements méthodologiques et idéologiques mis en oeuvre par les historiens, soit l'histoire-récit, l'histoire-tableau, l'histoire philosophique, l'histoire-problème et l'histoire structurale ou sociologique, l'histoire-problème paraît la plus appropriée pour produire une représentation du passé historiquement pertinente et scientifiquement valide. Les conceptions méthodologiques et idéologiques des premiers historiens du mouvement ouvrier français sont explicitées et analysées dans la perspective de démontrer la valeur épistémologique de l'histoire-problème. 


\title{
ARTICLES
}

\section{CRITIQUE DE LA PENSÉE HISTORIQUE \\ Les premiers historiens français du mouvement ouvrier* par Guy Massicotte}

\begin{abstract}
RÉSUMÉ. L'historien a pour fonction de reconstituer le passé de manière à conjuguer les exigences de rigueur méthodologique et de pertinence par rapport aux questions qui intéressent les hommes de son temps. Parmi les divers agencements méthodologiques et idéologiques mis en ouvre par les historiens, soit l'histoire-récit, l'histoire-tableau, l'histoire philosophique, l'histoire-problème et l'histoire structurale ou sociologique, l'histoire-problème paraît la plus appropriée pour produire une représentation du passé historiquement pertinente et scientifiquement valide. Les conceptions méthodologiques et idéologiques des premiers historiens du mouvement ouvrier français sont explicitées et analysées dans la perspective de démontrer la valeur épistémologique de l'histoire-problème.
\end{abstract}

ABSTRACT. Historians have to elaborate scientific representations of the past which are meaningful for the present. Among the various methodological and ideological conceptions used by them, that is narrative history, descriptive history, philosophical history, "problematic history " (histoire-problème) and structural or sociological history, "problematic history» seems to be the most appropriate. We intend in this study to explicate the conceptions underlying the first historiography of the French working movement, and to demonstrate the epistemological value of "problematic history" which combines scientific requirements and social relevance.

\section{INTRODUCTION}

L'historien réécrit le passé en fonction du présent. C'est là un lieu commun qu'un philosophe comme Adam Schaff a bien

* L'auteur remercie le Conseil des Arts du Canada pour son appui financier. 
illustré en montrant comment l'histoire d'un même objet, en l'occurrence la Révolution française, s'est modifiée au cours des années, selon, justement, que telles ou telles préoccupations contemporaines nouvelles apparaissaient et commandaient une nouvelle représentation du passé. ${ }^{1}$

L'historien part d'a priori méthodologiques et idéologiques que lui dictent le milieu dans lequel il vit et sa propre situation au sein de ce milieu. C'est même l'adéquation de sa vision du passé avec les préoccupations de ses contemporains qui fait toute la valeur et la pertinence d'une œuvre historique. Lucien Febvre l'a très bien démontré : cette condition propre au métier d'historien ne doit pas être considerée comme une faiblesse, elle donne au contraire toute sa valeur à l'histoire, dont la fonction, précisément, est de reconstituer le passé en fonction de problèmes qui se posent à l'homme dans son cheminement dans le temps. ${ }^{2}$ Certes, à mesure que notre vision du monde devient de plus en plus scientifique, davantage imprégnée de conceptualisations et davantage exigeante en matière de rigueur méthodologique, l'historien doit analyser et interpréter le passé en tenant compte de ces nouvelles exigences, mais sans perdre de vue le sens profond de son activité : le retour sur le passé en fonction du présent et de l'avenir, d'un avenir qui en aucun cas ne saurait obéir à une vision pré-déterminée, fût-elle à prétention scientifique.

Cette double exigence d'objectivité, de scientificité, et d'articulation avec la vision du monde des hommes du temps présent, s'est le mieux réalisée avec l'histoire-problème. On peut le voir lorsqu'on examine dans un corpus historiographique donné, l'articulation des a priori méthodologiques et idéologiques découlant de l'existence historique propre des historiens, avec les objets de leurs études. La production des premiers historiens du mouvement ouvrier français paraît particulièrement indiquée pour analyser les modalités de cette articulation et pour montrer la pertinence de l'histoire-

1. Adam Schaff, Histoire et vérité. Essai sur l'objectivité de la connaissance historique (Paris Anthropos (s.d.). 352 p.). On lira aussi pour le Québec: Serge. Gagnon, Le Québec et ses bistoriens de 1840 à 1920. La Nounelle-France de Garneau à Groulx (Québec: P.U.L., 1978. 474 p.).

2. Lucien Febvre, Combats pour l'histoire (Paris : Colin, 1965 (1953). ix-458 p.). 
problème comme genre historique capable de promouvoir l'objectivité sans trahir la mission propre de l'histoire. En effet, toute histoire qui s'écrit pour la première fois doit s'établir en marge, à l'encontre parfois, de la tradition, surtout si elle met en scène des mouvements historiques eux-mêmes marginaux ou en rupture de société. Comment croire, dès lors, qu'une telle histoire ne porte pas l'empreinte des préoccupations de ceux qui l'écrivent, ne traduise pas l'intense nécessité de se doter d'un passé, c'est-à-dire de racines capables de conférer un sens à la marche du temps, donc significatives pour l'avenir ? Si une historiographie bien constituée peut donner lieu à des démarches plus gratuites où l'historien a le loisir, à la limite, de se laisser prendre par des problèmes qui n'ont de sens qu'à l'intérieur du débat historiographique, il n'en est pas ainsi à la naissance d'un courant nouveau, où ceux qui écrivent sont des militants ou des hommes d'action à la retraite qui se donnent pour mission de consigner l'expérience vécue. En outre, l'époque qui a vu apparaître les premiers textes d'histoire ouvrière, soit la fin du XIX $\mathrm{X}^{\mathrm{e}}$ siècle et le début du $\mathrm{XX}^{\mathrm{e}}$ siècle, est aussi celle où toutes les forces de la culture cherchent à faire accéder l'histoire au statut de science, celle où l'on parle avec le plus d'abondance de méthode historique, et de rigueur, sinon d'objectivité, de la connaissance historique. ${ }^{3}$

Pour réaliser cette analyse, nous mettons en œuvre une typologie déjà utilisée pour l'œuvre de $\mathrm{Febvre}^{4}$ et pour celle des historiens plus récents du mouvement ouvrier français. ${ }^{5}$ Certes, les catégories d'histoire-récit et d'histoire-tableau, d'histoire-problème et d'histoire structurale, sont ici moins explicitement présentes que dans l'historiographie postérieure, mais le plus remarquable est de les retrouver dès cette époque, avec quelques variantes, sous-jacentes à l'organisation de la

3. La littérature sur l'histoire de l'histoire n'est pas très abonóante. Et dans ce sens, notre étude n'est pas de celles qui illustrent sur un point précis des thèses générales qui seraient bien connues. Elle voudrait plutôt, par une étude de détail élaborée dans une perspective toute particulière, contribuer à la constitution d'une interprétation d'ensemble de l'historiographie française. On verra tout de même pour la période en cause : William R. Keylor, Academy and Community. The Foundation of the French Historical Profession (Cambridge : Harvard University Press, 1875. x-286 p.).

4. Libistoire-problème. La méthode de Lucien Febvre (St-Hyacinthe : Edisem, 1981. 121 p.).

5. "Historiographie française de la question ouvrière : Réflexion de mérhode. La théorie de la connaissance dans quelques ouvrages récents". Histoire sociale, XI (21) (1978) $(183-214)$ 
production historique. Par ailleurs, nous essayons de pousser plus loin dans cette analyse l'explicitation des liens entre les aspects méthodologiques et plus proprement idéologiques qui existent à l'intérieur de ces catégories.

Nous avons ainsi identifié quatre (4) catégories d'agencement d'a priori méthodologiques et idéologiques structurant la matière historique concrète :

1) histoire-récit et histoire-tableau, assorties d'une vision positiviste et libérale de l'histoire. Les historiens qui pratiquent ce genre passent de l'histoire-récit à l'histoire-tableau pour décrire certains éléments qui ne se prêtent pas au mode narratif : contexte institutionnel, économique, social, culturel, mental. . à l'intérieur duquel prennent place les événements du récit; il s'agit d'une description de la réalité à un moment donné du temps, sans perspective évolutive, et qui se veut la plus factuelle possible ;

2) histoire-récit et histoire philosophique, où les personnages deviennent des entités collectives, idéologiques ou sociales ;

3) histoire-récit et histoire-problème, où le problème éclate au cœur du récit, et où la vision de la réalité est fonction du problème envisagé, ce qui laisse place à une très grande diversité d'options philosophiques au plan du choix et de l'importance relative des éléments historiques à mettre en lumière ;

4) histoire sociologique, ou quand la sociologie se met à l'école de l'histoire, ce qui annonce en fait l'histoire structurale et néo-positiviste; le modèle y structure l'œuvre, ce qui laisse la possibilité de privilégier telle ou telle dimension du réel ou telle ou telle option philosophique.

Au lieu d'expliciter plus longtemps dans l'abstrait les propriétés de chacune de ces catégories, nous allons analyser la pensée des auteurs qui se rattachent à chacune d'elles à travers les textes qu'ils ont écrits et que nous avons étudiés. 


\section{HISTOIRE-RÉCIT, HISTOIRE-TABLEAU, HISTOIRE POSITIVISTE}

Parmi les dix ouvrages que nous avons sélectionnés pour cet article, deux se rattachent sans conteste à cette catégorie et présentent par ailleurs des caractéristiques très intéressantes: le premier, parce qu'il incarne dans ce qu'elle a de plus pur la tradition érudite et positiviste $;^{6}$ l'autre, parce qu'il représente une tentative fort honnête d'appliquer la méthode de l'histoirerécit aux nouveaux objets mis en cause par l'histoire économique et sociale. $^{7}$

Léon de Seilhac, dans son livre consacré aux Congrès ouvriers, affiche, dès le point de départ, sa préoccupation positiviste et érudite, pour ne pas dire son fétichisme du document :

Il nous eût été facile d'écrire une brève histoire du mouvement ouvrier de 1876 à 1898 , mais combien de documents n'aurions-nous pas dû laisser de côté, par crainte d'alourdir la narration! Il nous a semblé préférable de laisser la parole aux rédacteurs analytiques de ces assemblées souvent mal ordonnées, quitte à résumer plus tard en une courte brochure, les faits saillants de cette période d'organisation, en renvoyant le lecteur à ce recueil de documents authentiques. ${ }^{8}$

Il s'agit donc d'un recueil de documents, d'un résumé des «Protocoles » comme dit de Seilhac, et c'est bien là, en effet, que conduit une conception de l'histoire rivée à l'ordre chronologique et qui prétend reconstituer la totalité du réel à partir des sources documentaires.

De Seilhac, bien entendu, fait précéder sa publication de documents d'une introduction qui dénote plus de largeur de vue, lorsqu'il explique, par exemple, comment on en est venu à instituer des Congrès. Mais il ne s'agit là que de quelques pages, sorte de hors d'œuvre, avant la retranscription des morceaux choisis qu'il a tirés des archives. Est-ce à dire que cette forme d'histoire possède, sinon le mérite de l'intérêt et de la hauteur de vue, tout au moins l'avantage de l'impartialité et

6. Léon de Seilhac, Les Congrès ouvriers en France (1876-1897) (Paris : Colin, 1899. 364 p.).

7. Émile Levasseur, Questions ouvrières et industrielles en France sous la Troisième République (Paris : Rousseau, 1907. 1xxii-968 p.).

8. De Seilhac, op. cit., v. 
de la vérité ? Elle le donne à penser en laissant la parole aux sources, mais s'il faut en croire Léon Blum, qui s'est lui-même livré à l'étude du même sujet, Seilhac errerait à plusieurs endroits dans le rapport des décisions et des notes. ${ }^{9}$ Et en outre, ce serait dans un sens bien particulier qui tendrait à diminuer l'importance de la tendance collectiviste au sein des Congrès! Voilà qui montre bien que même l'effacement le plus total de l'historien ne le met pas à l'abri, sinon de la partialité, tout au moins des accusations de partisannerie.

Avec Émile Levasseur, nous avons affaire à un tout autre genre d'historien, de très haut calibre, et remarquable à plus d'un point de vue: (1) il fut l'un des pionniers de l'histoire économique et sociale en France ; (2) il pratique l'histoire-récit et l'histoire-tableau, dont il justifie la valeur tout en étant conscient de ses limites quant à la scientificité et à l'objectivité ; (3) tout en privilégiant l'exposé factuel, il ne craint pas les jugements de valeur dont il explicite et légitime la teneur.

Un historien économique. L'ouvrage que nous analysons ici vient au terme d'un demi siècle de recherche et fut précédé d'au moins deux autres ouvrages du même genre, l'un pour la période pré-révolutionnaire et l'autre pour la période allant de la Révolution à la $\mathrm{III}^{\mathrm{e}}$ République. ${ }^{10}$ Celui-ci couvre la période 1870-1900. ${ }^{11} \mathrm{La}$ façon dont Levasseur explique l'avènement de l'histoire économique dans les préoccupations des historiens, mérite d'être citée :

Sous la Restauration et sous le gouvernement de Juillet, des historiens de renom, Guizot, Mignet, Augustin Thierry s'étaient attachés surtout à l'histoire politique et avaient décrit les institutions, les luttes, les progrès de la bourgeoisie dont l'avènement au pouvoir leur paraissait le couronnement de la civilisation. Déjà, cependant, les travaux des chartistes commençaient, avec Guérard et autres, à mettre au jour la vie populaire et tout d'abord la vie du paysan sous le régime

9. Léon Blum, Les Congrès ouvriers et socialistes franģais (Paris : Soc. nouvelle de librairie et d'édition, 1901. 4 vol.) : par exemple vol. I, p. 50.

10. Émile Levasseur, Histoire des classes ouvrières en France depuis lat conquête de Jules César jusqu'à la Révolution (Paris: Librairie de Guillaumin, 1859. 2 vol.); Histoire des classes ouvrières et de l'industrie en France, de 1789 à 1870 (Paris : Rousseau, 1903-1904 (1867). 2 vol.).

11. Émile Levasseur, Questions ouvrières et industrielles en France sou: la Troisième République (Paris: Rousseau, 1907. 1xxii - 968 p.). 
féodal. Dans la seconde moitié du $\mathrm{XIX}^{\mathrm{e}}$ siècle, le suffrage universel et l'éclosion des théories sociales pendant la seconde République attirèrent davantage l'attention sur la condition des travailleurs dont les œuvres, pour modestes qu'elles soient, forment le fonds principal de la vie sociale. L'histoire économique fut plus étudiée. J'ai été du nombre de ceux qui se sont adonnés à cette étude. $\grave{A}$ toute époque, les historiens ont reflété plus ou moins les préoccupations de leur temps. ${ }^{12}$

Les études précédentes de Levasseur portaient spécifiquement le titre d'Histoire des classes ouvrières, mais traitaient en réalité de l'ensemble de l'économie industrielle. «Sous la dénomination de Classes ouvrières, nous dit Levasseur, je comprenais tous les travailleurs de l'industrie de quelque ordre et condition qu'ils fussent, patrons, artisans, employés, ouvriers, apprentis », et dans ces ouvrages « je m'étais appliqué à exposer non seulement l'organisation du travail et les rapports des travailleurs entre eux, mais aussi les résultats du travail, le produit à côté du producteur, parce que l'un explique en partie l'autre ". ${ }^{13}$ Son troisième ouvrage, que nous examinons ici, est par contre davantage centré sur les questions du travail au sens contemporain du terme, et selon Levasseur, c'est la nature même de l'objet qui veut cette orientation. En effet, si «le privilège et la réglementation ont été la caractéristique dominante de l'organisation industrielle avant 1789 » et « la liberté secondée par la science. . . la caractéristique de la période 1789-1870. . ., la période contemporaine depuis 1870 sera plus particulièrement caractérisée par l'étude des problèmes concernant l'organisation des travailleurs et la répartition de la richesse $» .14$

L'introduction du mot "étude » nous rappelle que nous avons de tout objet historique une vision qui découle d'une étude, ce qui pose le problème du genre de cette étude, du mode d'expression choisi par un historien pour s'exprimer.

Histoire-récit et histoire-tableau. Levasseur croit aux vertus de l'histoire-récit, genre qu'il a lui-même pratiqué dans ses œuvres antérieures: «Quand l'historien peut embrasser

12. Levasseur, op. cit., $\mathbf{v}$.

13. Levasseur, op. cit., vi.

14. Levasseur, op. cit., vi-vii. 
dans la suite des temps une longue période, il aperçoit les faits dans une perspective qui l'aide à saisir le rapport des principales lignes; le recul contribue à faire voir les choses à leur place et dans leurs proportions ; car les causes ont produit leurs effets qui déposent pour ou contre. Il appartient à la clairvoyance de l'écrivain de discerner les uns et les autres. " ${ }^{15}$ Pour les périodes plus récentes, "les conditions sont moins bien favorables. On est dans la mêlée, trop près des détails pour discerner nettement les traits dominants. II faudra l'aide du temps pour que ceux-ci se dégagent de la multiplicité des traits secondaires, divergents et confus, et pour qu'ils apparaissent bien en lumière pendant que le reste s'estompera à distance. "16 Il ne faudrait pas croire, toutefois, que pour Levasseur la possibilité de l'objectivité soit liée à l'éloignement dans le temps de l'observateur et des faits observés. "Les écrivains qui traitent du passé sont-ils eux-mêmes toujours assez dégagés des préjugés du présent pour ne pas se laisser influencer par eux dans la manière de présenter les événements et dans les jugements qu'ils portent. . . Tel cui croit écrire l'histoire, voire même l'histoire grecque ou romaine, soutient, à son insu peut-être, une thèse fondée sur son interprétation personnelle des données documentaires. " ${ }^{17}$

C'est là, en fait, une condition inaltérable du métier d'historien que Levasseur énonce d'ailleurs très bien : " Je dois donc reconnaitre que les historiens, quelque souci qu'ils aient de la vérité et quelque époque qu'ils envisagent, se placent, involontairement sans doute, mais conformément à la tendance de leur esprit, à des points de vue divers pour observer le champ historique et qu'ils aperçoivent ainsi sous des angles différents des images différentes du même objet. " ${ }^{18}$ Ce qui ne veut pas dire, naturellement, qu'il faille confondre l'histoire avec le pamphlet : "Le pamphlétaire soutient délibérément une thèse. L'historien fait profession d'impartialité ; mais il n'abdique pas pour cela sa personnalité. " ${ }^{19}$

15. Levasseur, op. cit., vii.

16. Levasseur, op. cit., vii.

17. Levasseur, op. cit., vii.

18. Levasseur, op. cit., viii.

19. Levasseur, op. cit., viii ; l'auteur s'est très bien expliqué sur les rapports de ['histoire aver la science et plus précisément de l'histoire économique avec la science économique : 
L'histoire doit rester sur le terrain des faits, qu'elle narre selon l'ordre chronologique. Ce n'est pas cet ordre, toutefois, que Levasseur a choisi, pour cette dernière période, estimant sans doute que la contemporanéité interdisait la perspective chronologique, «historique », dit-il, comme par un abus de langage. Son étude reste néanmoins purement descriptive et réalisée selon le même procédé, « qui est celui de l'érudition et qui consiste dans la recherche et l'étude consciencieuse des documents originaux $»$.

«Mais, ajoute-t-il, puisque je renonçais à disposer les choses dans la perspective historique, j'ai dû les faire entrer dans un autre cadre. ${ }^{20} \mathrm{La}$ chronologie, en effet, est un support utile, pour l'historien metteur en scène de faits. Que se passe-t-il quand il y renonce?

J'ai adopté un cadre à compartiments. Chaque matière est exposée l'une après l'autre dans une suite de chapitres dont l'ordre et l'enchaînement ne paraissent pas au premier abord rigoureux. C'est en quelque sorte une composition à tiroirs. Ce n'est pas pour cela une marqueterie placée au hasard.

Essayant de donner une image aussi complète que possible de l'état du travail industriel en France et de la condition légale, matérielle, morale des travailleurs, j'ai composé une image à facettes, reflétant les divers aspects de la vie économique. ${ }^{21}$

C'est ce qu'on appelle l'histoire-tableau qui s'inscrit dans la même logique que l'histoire-récit, mais en substituant au cadre chronologique un cadre formel établi a priori. ${ }^{22}$ Cette conception de l'histoire, derrière son souci d'exhaustivité, n'est pas exempte, comme le voit bien Levasseur, d'une certaine

"L'histoire économique qui expose des faits n'est pas la science économique qui démontre des principes. Étant narratrice et non dogmatique, elle ne saurait avoir la prétention de se substituer à la science dont le rôle est d'observer, d'analyser et de comparer des phénomènes pour en dégager des lois qui les régissent [. . .] Elle l'est aussi parce qu'elle éclaire la science économique en faisant apercevoir, par un exposé chronologique des événements et de leurs conséquences, l'évolution dont la notion élargit et assouplit les théorèmes abstraits. L'histoire économique et la science économique se donnent la main et se complètent l'une l'autre. . . ".

20. Levasseur, op. cit. . $\mathbf{x}$.

21. Levasseur, op. cit., $\mathrm{x}$.

22. Pour une présentation théorique de cette conception, Charles V. Langlois ei Charles Se:gnobos, Introduction aux études bistoriques (Paris : Hachette, 1898 . xviii-303 p.) ; et pour une critique, Lucien Febvre, "Pour la synthèse contre l'histoire-tableau. Une histoire de la Russie moderne. Politique d'abord?" Combats pour l'bistoire (Paris : Colin, 1965 (1953). ix - 458 p.) (70-74). 
conception des choses, puisqu'il faut bien les ordonner d'une certaine façon. Ce qui pose le problème de sa philosophie de l'histoire, des jugements qu'il porte sur la réalité, des choix qu'il fait quant aux aspects à mettre en lumière.

Philosophie de l'histoire et jugement de valeur. Les faits en histoire s'ordonnent selon une certaine conception du réel qui trahit l'importance que l'historien accorde à telle ou telle dimension, au rôle ou à la fonction qu'il leur assigne dans le déroulement de l'histoire. C'est ce que l'on pourrait appeler la structure logique d'une conception de l'histoire. Cette présentation des faits s'accompagne de prises de position quant aux enjeux, aux significations, aux valeurs qui tissent la trame de l'histoire. L'historien ne prend pas seulement parti en présentant les choses d'une certaine façon, en valorisant l'importance de certains éléments, il prend aussi parti en privilégiant des interprétations, en donnant tort ou raison à des acteurs et à des idéologies. C'est ce que l'on pourrait appeler l'axiologie d'une conception de l'histoire. On retrouve, chez Levasseur, dans sa manière même de présenter les faits, une certaine conception de l'histoire, et mêlée à cette présentation, des jugements de valeur.

La première option de Levasseur à propos de la réalité historique concerne l'importance de l'économie :

Plus on étudie cette partie de l'histoire générale, plus on reconnaît non seulement qu'elle en est une partie considérable, mais qu'elle est étroitement liée aux autres par des relations de cause à effet, que les intérêts économiques exercent une puissante action sur l'ensemble des phénomènes sociaux et politiques d'une nation et que partant il est nécessaire de les connaître pour mieux comprendre l'histoire générale. Cette remarque, qui est vraie de tous les peuples et de tous les temps, l'est pour l'époque contemporaine plus que pour toute autre. ${ }^{23}$

Il opte ensuite en faveur d'une conception libérale de l'économie, dont la légitimité lui viendrait de son efficacité à développer les forces productives. Nous sommes ici sans aucun doute à la frontière de l'axiologie dans la mesure où la science et la liberté sont érigées en instruments de prospérité, et

23. Levasseur, op. cit., ix. 
hautement valorisées, de concert avec l'accroissement de la richesse, qui en est une conséquence :

Toutefois des inductions et déductions de la science, il ressort, entre autres vérités théoriques généralement reconnues, que la force productive de l'homme étant la source première de la création de la richesse, il importe au bien public que les forces productives humaines d'une nation soient le plus nombreuses et le plus développées possible, qu'elles soient le mieux secondées par l'abondance des capitaux fixes et circulants et par l'étendue et la précision de la technique et qu'elles jouissent par la concurrence de la plus grande liberté d'action compatible avec l'ordre social afin de produire tous leurs effers. Or, l'histoire économique du XIX ${ }^{\mathrm{e}}$ siècle confirme sur ce point la théorie en montrant par l'évidence des faits la grande influence qu'ont exercé sur l'accroissement de la richesse et par suite sur le bien-être des travailleurs, la liberté du travail, la plénitude de la propriété, l'invention dans les sciences mécaniques et physiques, le progrès de l'instruction, l'accroissement des capitaux, lesquels sont à la fois les produits, les soutiens et les stimulants de l'énergie humaine. ${ }^{24}$

De 1789 à 1870 , il ressort donc que « la liberté et la science ont été les deux grands principes générateurs du progrès » et que « l'accroissement du capital et l'importance des établissements industriels en ont été la conséquence et que ces diverses causes ont puissamment contribué à accroître la richesse ». Après 1871, les mêmes causes continuent d'agir, mais à celles-là s'en ajoutent d'autres : le suffrage universel et ses conséquences. "Il est aujourd'hui le souverain de la France, donnant aux masses populaires et particulièrement à la classe ouvrière des villes [. . .] une influence toujours croissante et désormais très puissante sur la direction des affaires, à côté de l'influence de l'argent. » Les relations entre le capital et le travail, la solidarité sociale au profit des classes défavorisées, et le socialisme qui pousse, en même temps que le syndicalisme, à la lutte des classes, sont devenus des questions de prime importance. ${ }^{25}$

On voit donc quelle conception de l'histoire anime Levasseur. Elle fait orchestrer les faits de la façon suivante.

24. Levasseur, op. cit., $\mathrm{x}$.

25. Levasseur, op, cit., $\mathrm{x}-\mathrm{xi}$. 
Tout d'abord, les choses : la production et la circulation des biens, le rôle joué par la science, les capitaux et les entreprises. Ensuite, les personnes: il parle "de leurs groupements professionnels, des idées économiques et sociales qui les dirigent, des lois destinées à régler leurs rapports comme travailleurs, des changements survenus dans la somme de leurs richesses et de leur condition matérielle et morale ". ${ }^{26}$

Cet exposé de la matière historique qui traduit une certaine conception du réel ne comprend pas seulement des faits exhibés et mis en valeur selon les conceptions de l'auteur, il comporte également des jugements de valeur au sens strict, qui suggèrent des lignes de conduite, des préférences quant à ce que devrait être l'avenir. Levasseur ne s'en cache pas, d'ailleurs. L'historien, dit-il, "s'il n'est pas simplement éditeur de textes, doit, après avoir mis en scène les faits et s'être en quelque sorte dissimulé derrière le théâtre, se faire, sobrement mais résolument, le juge de ces faits afin d'éclairer son lecteur et de l'inviter ainsi à penser par lui-même ". ${ }^{27}$ Dans sa préface, Levasseur expose les grandes lignes de sa philosophie basée essentiellement sur le libéralisme (liberté de penser et d'agir comme valeur intrinsèque et comme facteur créateur de richesse et de bien-être) et le rationalisme (recherche de la vérité, dans la mesure où la vérité scientifique concourt à la liberté et au bien-être).

Toutefois, c'est peut-être dans la manière dont il aborde les problèmes historiques proprement dits que l'on peut apercevoir avec le plus d'éclat quelle est sa philcsophie sociale. Prenons par exemple les syndicats et les grèves. Pour Levasseur, "les syndicats ouvriers ayant pour objet la défense des intérêts ouvriers, il est naturel qu'ils s'en soient servis pour défendre ces intérêts par la coalition quand ils se sont trouvés en conflit avec les intérêts des patrons et que ces syndicats aient en conséquence provoqué, organisé, soutenu des grèves ". Ce qui ne l'empêche pas d'estimer qu'elles "sont des agitations qui troublent sur le moment l'industrie et qui causent au patron et à l'ouvrier des pertes lentement recou-

26. Levasseur, op. cit., xii.

27. Levasseur, op. cit, viii. 
vrables, et dont le mal devient parfois irréparable quand la clientèle se déplace ou quand l'industrie s'atrophie $"{ }^{28}$ Comme il le dira dans l'épilogue : on ne pourra jamais répartir plus de richesses que l'on en crée; et en sous-entendu, les luttes pour le partage de la richesse nuisent à la croissance de celle-ci et desservent par conséquent ceux qu'elles prétendent servir. ${ }^{29}$ Mais ce qu'il déplore le plus, "c'est qu'aux intérêts réellement professionnels, pacifiquement discutés, se mêlent des préoccupations révolutionnaires et des haines systématiques de classe [. . .]». "Pour mainte grève, ajoute-t-il, la revendication professionnelle est une occasion de s'insurger contre l'organisation sociale, contre l'existence du patronat et de préparer par un bouleversement l'avènement d'une organisation socialiste du travail. C'est surtout dans ce cas que sont fréquents les désordres matériels, propriétés saccagées, personnes violentées, dont on cite tant d'exemples. " ${ }^{30}$ Comme on le voir, c'est là une conception toute libérale qui postule la valeur intrinsèque et universelle d'un système où se discute librement et pacifiquement la poursuite des intérêts de tous et de chacun, intérêts qui cohabitent d'ailleurs en toute solidarité ; cette conception est aussi empreinte de rationalisme, dans la mesure où la raison, c'est-à-dire l'atteinte du consensus par la convergence rationnelle des intérêts, est érigée en valeur suprême.

Levasseur est un historien de tendance positiviste. Certes, il ne croit pas que l'objectivité soit totalement possible, ni même souhaitable. Il estime que les conceptions propres de l'historien marquent sinon la description proprement dite des faits tout au moins le choix des aspects qu'il décide de mettre de l'avant, et que ces conceptions doivent même s'exprimer ouvertement sous forme de jugement de valeur une fois la description terminée. Néanmoins, il croit à la vérité et à la science en privilégiant au plus haut point le factuel et l'exhaustivité : son tableau de l'économie française en est un exemple éloquent. En outre, sa philosophie sociale éminemment libérale s'accorde avec son rationalisme scientiste, dans la

\footnotetext{
28. Levasseur, op. cit., 944 .

29. Levasseur, op. cit., 960-961.

30. Levasseur, op. cit., 944.
} 
mesure où l'importance qu'il accorde à la science et à la recherche de la vérité se justifie par leur apport décisif à la réalisation de la société idéale.

\section{HISTOIRE-RÉCIT ET HISTOIRE PHILOSOPHIQUE}

Levasseur ne raconte pas une histoire. Il décrit un système, en montrant ses fondements (liberté, science, capital, entreprises) et ses caractéristiques, et prend parti en faveur de certaines valeurs liées à ce système : la liberté d'agir et de penser, la recherche rationnelle de la vérité et de l'intérêt. D'autres historiens préferent raconter l'histoire, c'est-à-dire décrire l'évolution, la métamorphose, de groupes d'hommes, d'institutions ou de questions qui se sont posées dans l'histoire.

L'idéal de l'histoire-récit dans la conception positiviste est la description des faits, la mise en scène du réel à l'état pur, en prenant parti ça et là selon les conceptions propres de l'auteur, qui se réflètent en outre inévitablement dans le choix des objets mis en scène, dans les types de relations fonctionnelles ou causales privilégiées et dans les modulations d'emphase ou d'insistance au sein du récit. Alors que certains poussent la volonté d'effacement de l'historien et le souci d'exhaustivité jusqu'à réduire l'histoire à la publication de textes, d'autres acceptent plus volontiers la synthèse personnelle et l'immixtion du jugement de valeur. Les libéraux, naturellement, qui considèrent le système économique et social comme un donné, pratiquent plus volontiers l'histoire politique où les partis et les hommes publics sont perçus comme les acteurs uniques de la destinée humaine. Même quand ils abordent l'histoire économique et sociale - Levasseur en est un exemple - ils ne discernent pas de perspective historique, sinon pour anticiper plus de richesse et plus de connaissances : la société libérale et bourgeoise est pour eux définitivement installée.

La tradition de l'histoire-récit fut cependant prise en charge par le socialisme, pour qui, sur le plan de l'histoire économique et sociale, la classe ouvrière et le socialisme relayent la bourgeoisie et le libéralisme comme acteurs principaux. Et comme les premiers historiens bourgeois l'avaient fait (Guizot, Michelet, Tocqueville), les premiers 
historiens socialistes, ou socialisants, pratiquèrent volontiers une histoire plus philosophique et interprétative que descriptive et factuelle. ${ }^{31}$

Pour illustrer l'histoire-récit, philosophique et interprétative, du mouvement ouvrier français, nous avons retenu trois historiens qui représentent trois orientations de la philosophie de l'histoire à l'intérieur du même mode d'expression.

Kritsky, la thèse marxiste. L'ouvrage de Kritsky, L'évolution du syndicalisme en France, ${ }^{32}$ est un exemple typique de l'historiographie marxiste traditionnelle : un récit dont les personnages sont les classes, mues par leurs intérêts, un récit dont la structure et la trame, générées par les structures économiques, prévoient la victoire, c'est-à-dire la domination, du prolétariat sur la bourgeoisie.

Le récit s'amorce avec "Les origines du Prolétariat et ses premiers groupements" qui laisse peu de doutes sur la philosophie de l'histoire de l'auteur. Avec la Révolution française, "le vieux monde disparut, balayé par la tempête révolutionnaire, ouvrant une ère nouvelle du capitalisme, de la société moderne basée sur les intérêts bourgeois et non plus nobiliaires. Ce fut l'avènement de nouvelles couches sociales. " ${ }^{33}$ Voilà pour la structure de classes. Pour ce qui est de la lutte de classes qui va conduire au syndicalisme :

Le prolétariat, c'est cette classe d'individus juridiquement libres, qui n'ont pour ressource unique ou essentielle que le louage possible de leur travail aux propriétaires des moyens de production. Si donc en droit l'homme a cessé d'être soumis à l'homme, il ne cesse pas de l'être en fait.

Inaliénable en droit, le salarié devient une chose, un outil ; il est obligé de subir les conditions de crédit ou de travail qui lui sont offertes quelles qu'elles soient, sans même que la

31. Ce fut sous l'effet d'un positivisme qui niait à la connaissance historique la possibilité de la formalisation théorique, trahissant ainsi en quelque sorte sa conviction que l'évolution historique était parvenue à son terme, que l'histoire-récit dégénéra en un respect du fait concret, dévotieux, artificiel et bien souvent faux, dans la mesure où il justifiait une philosophie implicite de l'histoire (Lavisse, Rambaud, Langlois, Seignobos, . . .)

32. Mlle Kritsky, L'évolution du syndicalisme en France (Paris : Giard et Brière, 1908. iv 426 p.). L'ouvrage porte comme nom d'auteur "Mlle Kritsky " sans identification de prénom. Des recherches dans des guides bibliographiques n'ont pas permis de retrouver l'identité complète de l'auteur.

33. Kritsky, op. cit., 1-2. 
subsistance lui soit garantie par les détenteurs actuels des moyens de vivre.

Nous voyons d'un côté surgir l'autorité, les richesses, les jouissances, le luxe, les plaisirs ; de l'autre, toutes les charges de la société, l'incertitude du lendemain. ${ }^{34}$

À ce point d'ailleurs, la référence à Marx est explicite : "La société moderne, la société bourgeoise, née de l'écroulement de la société féodale, n'a pas aboli les antagonismes de classe. Elle n'a fait que substituer des classes nouvelles, de nouvelles possibilités d'oppression, de nouvelles formes de luttes à celles d'autrefois [...] De plus en plus la société entière se partage en deux grands camps ennemis, en deux grandes classes directement opposées : la bourgeoisie et le prolétariat. » ${ }^{35}$ «Mais, ajoute Kritsky, cet antagonisme n'apparait pas nettement au commencement. ${ }^{36}$ C'est au récit du développement de cet antagonisme qu'elle procède dans les pages qui suivent.

Les passages qu'elle consacre aux racines économiques de ce conflit sont particulièrement brillantes: l'essor de la production, le machinisme, la division et la dégradation du travail, les bas salaires, l'insécurité d'emploi en période de haute concurrence, la concentration industrielle . . .

Mais la société capitaliste, engendrant ces maux portait le remède en son sein. Le capitalisme, plaçant les ouvriers sur le même terrain économique, établissait une cohésion naturelle, mécanique d'abord, un lien plus étroit entre les milliers de salariés, agglomérés sur un espace restreint.

La même durée du travail, les mêmes souffrances, les mêmes inéquités éveillaient peu à peu chez les travailleurs leur conscience et les poussaient vers l'organisation. ${ }^{37}$

C'est ainsi que naissent les syndicats dont l'auteur retrace l'histoire dans les chapitres subséquents. Dans cette histoire plutôt institutionnelle, le lien reste vivant avec la société et sa structure de classes :

34. Kritsky, op. cit., 3-4.

35. Karl Marx et Friedrick Engels, Le Manifeste du parti communiste, 2i-22, cité par Kritsky, op. cit., 4.

36. Kritsky, op. cit., 4.

37. Kritsky, op. cit., 34 . 
Cependant, le prolétariat, en dépit de toutes ces vexations, se réveille, quelques corporations se réorganisent pour pouvoir résister aux. organisations patronales.

Découragés par l'échec formidable des révolutionnaires, intimidés par la répression sauvage, les travailleurs tournent leur espoir vers l'organisation sur le terrain pacifique, voyant dans l'association le remède à tous les maux. ${ }^{38}$

De même, la dimension téléologique de cette histoire reste toujours présente. Après la Commune, " le rôle de la bourgeoisie comme dépositaire du progrès fut moralement anéanti. Dès lors, c'est le prolétariat qui portera le drapeau du progrès et de l'émancipation de l'humanité. ${ }^{39}$ Sur le terrain de l'histoire concrète, la montée du syndicalisme et son effort de radicalisation et de concentration sont hautement prisés.

L'histoire-récit prétend jaillir du cœur même de l'histoire-vécue, surtout quand elle renonce à ses apparences d'objectivité factice qui consistent à ne retenir de la matière vivante de l'histoire que des traces scrupuleusement examinées jusqu'à leur complet desséchement, pour reconstituer le passé en fonction d'une idéologie profondément enracinée dans une fraction importante de l'esprit public, ou même d'une croyance empreinte d'émotions.

À mesure que l'histoire devient sociale, même quand elle demeure construite sur le modèle de l'histoire-récit, de nouveaux personnages font leur apparition: le syndicat, l'idéologie. Et comme, même à l'intérieur de l'histoire-récit, certains historiens assument très bien leur philosophie de l'histoire, nous avons droit à une interprétation explicite de la genèse et de l'évolution de ces institutions, de ces personnages de l'histoire sociale. Alors que Kritsky s'attachait au syndicalisme dans la perspective d'une interprétation rigidement marxiste, les deux historiens que nous allons maintenant considérer ont reconstitué le passé d'autres institutions de l'histoire sociale, en fonction de systèmes interprétatifs plus ouverts et plus critiques, qui les amènent dans la proximité même de l'histoire-problème.

38. Kritsky, op. cit., 118.

39. Kritsky, op. cit, 115. 
Paul Louis, genèse et nature du socialisme français. Dans son Histoire du socialisme français, Paul Louis traite du socialisme français, "qu'il ne faut pas confondre, dit-il, avec l'histoire des ouvriers en France ". ${ }^{40}$ Il veut montrer comment s'est constitué le prolétariat. "Comment est née l'idée d'une classe prolétarienne distincte, comment cette classe s'est comportée à travers les vicissitudes politiques et sociales survenues depuis la Révolution. " Mais surtout, selon les dires de sa préface, il désire avant tout s'attarder à "ce qui distingue le prolétariat de toutes les classes opprimées, qui l'ont précédé et qui se sont affranchies avant lui ", c'est-à-dire le fait "qu'il professe des théories tellement arrêtées que d'aucuns, par ironie, les ont qualifiées de clogmes [...] L'historien a pour mission de rechercher l'origine de ces thèses, leur expansion à travers le siècle, leurs relations avec le milieu économique, où elles évoluaient. " 41

Une telle façon de présenter l'ouvrage l'apparente à l'histoire-problème, mais ce n'est qu'une apparence: Paul Louis ne veut pas montrer comment et pourquoi le prolétariat en est venu à se doter d'une doctrine particulière, mais plutôt raconter la genèse et l'évolution de cette doctrine qui constitue le trait distinctif et une caractéristique essentielle du prolétariat. Telle est bien, en effet, la substance de l'ouvrage, qui n'est pas dépourvu par ailleurs d'intentions prosélytiques : " Il y a tout profit à ramasser, en un court écrit, les idées et les actes de trois ou quatre générations de socialistes. À ceux qui l'ignorent, cette histoire apprendra que le prolétariat possède une tradition déjà vieille; à ceux qui affectent de l'ignorer, elle le rappellera. " 42

Ce qui frappe le plus dans l'ouvrage de Louis, c'est une philosophie de l'histoire synthétique, où pourtant le monde des idées ne perd pas son autonomie relative. Les principaux courants de la pensée socialiste sont décrits en fonction des hommes qui les ont animés, des événements qui les ont vu naître et se propager, des structures auxquelles ils sont liés. Les

\footnotetext{
40. Paul Louis, Histoire du socialisme français (Paris : Éd. de la Revue Blanche, 1901. 313 p.): v.

41. Louis, op. cit., v-vi.

42. Louis, op. cit.. vi.
} 
penseurs et les idées socialistes s'insèrent dans tous les plis et replis de la société, sans perdre, toutefois, leur spécificité et leur autonomie. Les passages qui suivent montrent bien que Louis veut intégrer les idées dans le contexte des structures, des hommes et des événements, au demeurant, aussi bien politiques, économiques que culturels ou sociaux :

C'est dans cette étape de notre histoire, alors qu'entre le régime économique et le régime politique, le divorce se creusait chaque jour, quapparurent les précurseurs du socialisme, les écrivains que depuis lors, on appela les «utopistes ${ }^{4}$. 3

Plus on avance vers 1840 , et plus l'entente paraît étroite entre la petite bourgeoisie et le prolétariat : celle-là appelle celui-ci dans les sociétés secrètes, qui finissent par ne plus compter qu'une minorité de républicains politiques, et dont les manifestes se compliquent de plus en plus de déclarations à tendances socialistes. ${ }^{44}$

Or, la bourgeoisie gouvernante avait assez d'armes pour enrayer ce courant, dès sa naissance. La conquête des pouvoirs publics que nous attendons, plus ou moins, du rayonnement de nos théories, de l'activité de nos orateurs et de nos écrivains, et de la formation toujours plus disciplinée du prolétariat, était interdite aux hommes du règne de Louis-Philippe, s'ils s'en tenaient aux moyens strictement légaux. La légalité les tuait [... $]^{45}$

Il reste à dire pourquoi cette littérature [marxiste] est intervenue de préférence à l'étape où nous sommes arrivés. Elle a été déterminée comme il était naturel par l'état de l'évolution économique, par le relief même que les phénomènes nouveaux de la production acquéraient de jour en jour. C'est alors que sur toute la surface de l'Europe Occidentale, le machinisme déploie ses effets en toute leur plénitude [. . . $]^{46}$

Il ne faudrait pas prendre cette dernière phrase au pied de la lettre et croire que selon Paul Louis, les idées sont totalement déterminées par les infrastructures économiques:

L'évolution industrielle issue de la diffusion du machinisme, a pu renforcer d'arguments précis et prépondérants la dialectique communiste; elle n'a pas créé le communisme et cette seule

\footnotetext{
43. Louis, op. cit, 60 .

44. Louis, op. cit., 105.

45. Louis, op. cit., 110.

46. Louis, op. cit., 116.
} 
constatation a sa portée. Elle n'affaiblit pas, à coup sûr, la thèse matérialiste, mais elle suffit à montrer que $l \epsilon$ socialisme ne surgit pas tout entier de la transformation des modes de production, qu'il a été seulement corroboré et stimulé par elle, et qu'il répond tout aussi bien à des pures conceptions de l'esprit, aux catégories philosophiques d'équité et d'égalité sociale. Ainsi l'idéologie, en ce qu'elle offre de solide, se conciliera forcément, et à la lumière des faits historiques mêmes, avec le matérialisme. Celui-ci servira en quelque sorte de justification à celle-là, qui ne sera plus qu'une façon d'intuition et de prescience. ${ }^{47}$

Ainsi le monde des idées puise à ses propres sources, ce qui lui confere une certaine noblesse, une certaine force aussi, dans la mesure où, pertinent, il sait s'articuler au monde de la matière. Cette distanciation par rapport au matérialisme historique qui laisse une plus grande place à la pensée libre et critique, et qui s'enracine, par-delà sa vérité intrinsèque, dans l'incapacité de l'homme à se river sans espoir aux rivages de la matérialité, sert de matrice à l'histoire-problème. C'est dans le double refus du libéralisme qui idéalise le systèrne économique et social dans son état naturel, en permettant tout au plus, dans le cadre des finalités mêmes du système, des interventions humaines gouvernées par la science et la raison, et du matérialisme historique où l'esprit devient un produit de la matière, elle-même orientée dans un sens historique précis, que prend racine la possibilité même de l'histoire-problème. Mais avant d'examiner cette manière d'écrire l'histoire chez des historiens particuliers, il nous faut considérer un autre ouvrage dont la philosophie de l'histoire se déploie aux frontières de l'histoire-problème.

Daniel Halévy et ses Essais sur le mouvement ouvrier. Avec Daniel Halévy, nous restons roujours sur le terrain de l'histoire-récit, puisque c'est bien l'histoire des origines et de la croissance du mouvement ouvrier, de ses institutions, de ses manifestations, qu'il nous raconte dans ses Essais. Mais comme l'indique ce titre d' "Essais », il s'agit d'une œuvre interprétative, philosophique dans la mesure où c'est une conception de la réalité sociale qui est mise en œuvre à travers la description de son émergence dans le temps:

47. Louis, op. cit., 4. 
depuis la naissance sociale de l'ouvrier jusqu'à l'œuvre du militant qui prend en charge l'organisation ouvrière en vue d'influencer le déroulement de l'histoire.

«Ils ne savaient à fond aucun métier, et gagnaient leur vie en servant les machines. Ils étaient un embarras administratif. ${ }^{48} \mathrm{C}$ est le point de départ, le point zéro de l'histoire ouvrière. Plongés dans la misère que leur imposent les règles du jeu de la société industrielle naissante, les ouvriers doivent prendre en main leur propre destinée. « Les patrons n'entendirent pas l'appel que Robert Owen leur avait adressé. Il appartenait aux ouvriers anglais eux-mêmes de prendre en mains leur propre cause. Dès 1824, ils obtenaient le droit syndical, et pouvaient s'imposer à leurs maîtres par la force de l'organisation. ${ }^{49}$ Cette organisation qui s'incarnera tout d'abord dans le syndicat et ensuite dans le parti politique, est impossible sans le travail du militant. Halévy décrit la naissance sociale de ce personnage : " un homme mûr, de sens solide, et de caractère ferme, qui accepta de se mettre en avant, parce que, désigné par tous, il ne pouvait sans lâcheté se récuser. Il agit avec résolution, et sans joie : car il sait quel avenir inquiétant le guette, la mise à pied, l'inscription sur les listes noires [...] ${ }^{50} \mathrm{La}$ mobilisation des ouvriers autour de leur intérêt commun, l'œuvre du militant et la croissance de l'organisation syndicale s'épanouissent au sein d'une force qui les englobe: le socialisme. "Certes les syndicats sont loin d'être tous socialistes et ils se tiennent en dehors du mouvement politique, mais les ouvriers syndiqués subissent, parfois sans même le savoir, l'influence des révolutionnaires. C'est parmi eux que les militants du Parti vont chercher le public de leurs réunions, les recrues de leurs groupes: le syndicat est l'école primaire du socialisme. ${ }^{51}$

Le récit de ce processus particulier de la naissance du mouvement ouvrier s'élève tout de même à un certain niveau de généralisation quand Halévy décrit des scénarios types qui

48. Daniel Halévy, Essais sur le mouvement outrier en France (Paris : Société nouvelle de librairie et d'édition, 1901. 300 p.) : 3 .

49. Halévy, op. cit., 33.

50. Halévy, op. cit., 58.

51. Halévy, op. cit., 75-76. 
se reproduisent ici ou là dans le temps ou l'espace. Quand il parle, par exemple, de la transformation du métier de militant ou de meneur :

Par métiers et par régions les syndicats vont s'unir, et, riches
de leurs cotisations additionnées, ils pourront salarier un des
leurs qui deviendra secrétaire et représentant d'une fédération.
Jadis ouvrier, il aura été choisi entre dix ou vingt concurrents,
et la sélection se fera nécessairement dans le sens le plus sage,
car il ne s'agit plus, pour administrer une organisation
permanente, d'avoir ces dons de véhémence qui peuvent un
instant égarer une foule. Il faut savoir écrire, compter,
discuter ; il faut connaitre à fond un métier, ses machines, ses
matières premières, c'est-à-dire posséder une véritable science,
et les vertus les plus opposées à la démagogie.

Ce genre de constatations que nous retrouvons à plusieurs endroits dans l'œuvre d'Halévy élève le récit au-dessus du niveau de la poussière du fait unique et concret où prétendaient vouloir le maintenir les historiens positivistes, même quand ils admettaient comme Levasseur l'immixtion du jugement personnel de l'historien. Celui-ci devient un fait historique, et non plus seulement le fait de l'historien greffé sur la trame de l'histoire.

La différence la plus importante, toutefois, entre l'histoire-récit des positivistes et celle plus philosophique de ces historiens réside précisément dans la nature même de leur philosophie de l'histoire: marxiste (Kritsky), socialiste (P. Louis), social-démocrate (D. Halévy). Alors que les premiers finiront par tenter désespérément de dissimuler leur philosophie de l'histoire, conservatrice et desséchante, car elle souhaite au fond voir l'histoire mourir plutôt que continuer sa marche dans un sens qu'elle réprouve, les seconds prennent pour objet de leur préoccupation la réalité sociale dans son devenir et se la représentent selon une conception qui donne corps au récit de l'avènement de ce devenir:. Chez Daniel Halévy, nous retrouvons une philosophie de l'histoire synthétique, qui intègre plusieurs dimensions de la vie collective, et indéterministe, parce qu'elle laisse l'homme responsable et libre de son avenir. C'est ainsi que la trame de la naissance des

52. Halévy, op. cit., 59. 
institutions sociales est constamment mise en relation avec la vie économique et politique. À titre d'exemple, ce commentaire :

L'essor industriel qui marque la décade 1780-1790 précipita le mouvement. Les inventions de procédés, la mobilité des prix accrue par l'extension du marché international, achevèrent d'ébranler les vieux règlements, que la constituante abolit d'un vote.

La désorganisation est alors accomplie. La machine a triomphé des petites barrières qui limitaient son action, et c'est elle qui va profiter de la crise qu'elle a déterminée, enrichissant ceux qui la possèdent, et réduisant le peuple au métier de manœuvre. ${ }^{53}$

Mais c'est quand le récit d'Halévy devient normatif qu'il met en relief de la façon la plus manifeste la spécificité de sa philosophie de l'histoire, ouverte et indéterministe. Halévy blâme les conservateurs pour leur opposition au mouvement syndical, opposition qu'il estime dangereuse : "L'humanité est une lourde masse, souvent brutale et déplaisante ; mais sa lourdeur même est une garantie de bon sens. Elle ne se met en branle que rarement et pesamment, sous l'action d'un extrême besoin. Façonnée par la misère, elle est peu exigeante, et quand elle se plaint, il faut l'écouter : le danger est dans les obstacles qu'on lui oppose et qu'elle doit ensuite briser pour se frayer un passage. ${ }^{54} \mathrm{Il}$ est nécessaire, cependant, que ces revendications soient réfléchies et réalistes : «Or, aucune institution ne saurait, mieux que le syndicat, guérir les militants révolutionnaires du mal démagogique [...] Le syndicat mène au socialisme les ouvriers modérés; et il mène les socialistes à la modération, ou, pour mieux dire, à la réflexion. " $" 55$ Rien n'est donné d'une façon absolue : l'ouvrier se façonne au gré des attitudes et des décisions et plus elles sont éclairées et réfléchies, plus l'avenir sera harmonieux et civilisé. Nous voici dès lors tout proche de la philosophie de l'histoire qui s'exprime dans l'histoire-problème.

53. Halévy, op. cit., 4-5.

54. Halévy, op. cit., 76.

55. Halévy, op. cit., 76. 


\section{HISTOIRE-RÉCIT ET HISTOIRE-PROBLÈME}

Avant 1914, il serait illusoire de chercher des historiens qui auraient pratiqué l'histoire-problème, genre qui ne fleurira que plus tard et dont Lucien Febvre sera le théoricien et sans doute le plus grand praticien. Ce que nous apercevons pour l'heure, c'est une métamorphose du récit qui s'élève au questionnement, embrassant le réel dans sa diversité tout en restant le plus ouvert possible par rapport aux options philosophiques susceptibles d'intervenir dans le choix et l'interprétation d'un phénomène historique. Nous avons retenu quatre ouvrages qui illustrent chacun à sa manière cette façon d'écrire l'histoire. ${ }^{56}$

A. Souchon et la crise de la main d'œuvre agricole. ${ }^{57}$ La France s'est débattue pendant les 20 dernières années du XIX ${ }^{\text {ième }}$ siècle avec une crise agricole qui parâtt s'être résorbée depuis le début du $\mathrm{XX}^{\text {ième }}$ siècle. Mais ce ne sont là que des apparences : "Et de redoutables difficultés se dressent [. . .] dont on peut dire sans exagération qu'elles sont une crise nouvelle. C'est une crise de main d'œuvre. Elle est constituée par deux ordres de fait $»:$ d'une part les campagnes se vident, de l'autre, les rapports entre employeurs et salariés s'enveniment. «La preuve en est dans l'éclosion sur bien des points du territoire de syndicats rouges résolument hostiles au patronat, et derrière ces syndicats dans la multiplication des grèves agricoles. "Jusqu'ici, selon Souchon, on a beaucoup étudié le dépeuplement des campagnes. "Par contre, les syndicats et les grèves d'ouvriers agricoles [...] n'ont donné lieu qu'à des travaux fragmentaires, et d'ailleurs relativement peu nombreux. » Le but de l'auteur est donc de « reprendre, dans son ensemble, l'étude de la main d'œuvre agricole en France », en insistant davantage « sur les syndicats et les grèves ". ${ }^{58}$

C'est là une des caractéristiques les plus importantes de l'histoire-problème que de bien camper le sujet, un sujet qui

56. Un autre ouvrage aurait pu être analysé sous cette rubrique: Hubert Lagardelle, L'évolution des syndicats ourriers en France. De l'interdiction à l'obligation (Thèse soutenue à ta Sorbonne, le 9 novembre 1901).

57. A. Souchon, La crise de la main d'autre agricole en France (Paris : Rousseau, 1914. 552 p.).

58. Souchon, op. cit., $1-2$. 
doit répondre par ailleurs à des préoccupations qui s'enracinent de quelque façon dans l'esprit du temps. Mais il n'y a pas que le choix du sujet, il y a la façon de le traiter aussi. A cet égard également, le travail de Souchon annonce l'histoire-problème. En examinant la situation dans les diverses provinces, écrit-il, " nous apprendrons à connaître une unité de doctrine et presque d'action avec les efforts qui aspirent à grouper tout notre prolétariat agricole en une vaste fédération, filiale terrienne de la C.G.T. ". " Mais, ajoute-t-il, tout ne saurait être dans l'examen des faits. Nous aurons à en chercher les causes. ${ }^{59}$

Déjà en choisissant d'étudier une "crise ", Souchon ouvrait la voie vers un récit plus problématique que s'il eût décrit une institution dans son devenir ; en voulant, en outre, chercher les causes de cette crise, il fait un pas de plus, dans la mesure où le principe d'organisation de son texte ne sera plus seulement descriptif, mais aussi, explicatif, par rapport à l'objet qu'il veut décrire. Non sans réticence, il va jusqu'à énoncer en introduction l'hypothèse qui le guidera. "Le moment n'est pas, dans une introduction, d'indiquer les conclusions auxquelles nous serons conduits, le chemin une fois parcouru. Mais une remarque d'ordre général s'impose dès maintenant. ${ }^{60}$ Quelle est cette considération ? Il introduit tout d'abord une distinction entre la crise de mévente de la fin du XIXième siècle et la présente crise de main d'œuvre. Contrairement à la première, dit-il, celle-ci ne s'explique pas par des seules causes économiques. Les difficultés de main d'œuvre ont des causes économiques bien sûr, l'augmentation du bien-être matériel fait baisser la natalité et les salaires plus élevés dans les villes attirent les agriculteurs. "Mais, par ailleurs, dans l'ensemble des questions que nous allons étudier, nous trouverons au premier plan, des éléments d'ordre social et par conséquent d'ordre moral. "Si les ruraux se laissent si facilement séduire par les salaires industriels, c'est que rien ne les retient, "toutes les forces de tradition et de moralité capables de leur faire aimer la vie rurale ayant été volontairement détruites ». Ce facteur joue également dans la baisse de

59. Souchon, op. cit., 2-3.

60. Souchon, op. cit. 3 . 
la natalité et, ajoute-t-il, " ce n'est pas davantage dans l'ordre matériel qu'il faut chercher les raisons des moins bons rapports entre le prolétariat agricole et ses patrons. ${ }^{61}$

Comme on le voit, les explications sont recherchées à tous les niveaux de la réalité sociale et l'interprétation joue sur plusieurs registres sans privilégier d'emblée le déterminisme économique ou le leadership politique ou moral. Il s'agit bien là d'une caractéristique propre à l'histoire-problème et qu'il faut relier à cette autre particularité : que l'histoire doit servir à éclairer notre compréhension et à prendre parti d'une manière plus pertinente dans les problèmes de l'existence. Et c'est là une préoccupation qui anime Souchon. Parce que la crise n'est pas seulement économique, dit-il, elle ne se résorbera pas d'elle-même : ses causes sont tellement générales qu'elle risque même d'aller en s'aggravant. Dans cette perspective, "notre étude, estime-t-il, nous montrera ce qu'on peut espérer, ne fût-ce qu'en s'engageant résolument dans la voie qui améliorera les logements des domestiques [. . . N Nous verrons aussi tout ce qu'il est permis d'attendre des syndicats où les ouvriers seront avec leurs patrons réunis pour la défense d'intérêts communs, de bien d'autres mesures encore. C'est assez pour ne pas vouloir et ne pas mériter le reproche d'un pessimisme systématique. " ${ }^{62}$

L'étude de Souchon n'est pas l'œuvre d'un historien, même si son sujet et sa démarche sont essentiellement historiques. Les historiens reconnus comme tels à cette époque se sont de toute façon assez peu préoccupés de la question ouvrière, comme il est d'ailleurs dans l'habitude des historiens professionnels de négliger les phénomènes récents. Et quand ils l'ont fait, c'est par le biais d'une approche plus traditionnelle : à preuve, cette biographie de P.J. Proudhon par Édouard Droz, professeur à la Faculté des lettres de l'Université de Besançon. ${ }^{63}$

Proudhon vu par E. Droz. Même si cet ouvrage est une biographie et encore celle d'un théoricien et d'un militant

61. Souchon, op. cit., 3-4.

62. Souchon, op. cit., 5 .

63. Édouard Droz, P.J. Proudbon 1809-1865 (Paris: Pages libres, 1909. 284 p.). 
politique plus que d'un militant ouvrier comme tel, il méritait d'être signalé car c'est un livre à thèse qui ne paraît pas s'inspirer d'une philosophie de l'histoire bien déterminée. Certes, il n'est pas question ici d'une hypothèse qui se transforme en conclusion. La démonstration est tout entière construite sur le mode affirmatif. Néanmoins, parce que l'auteur veut démontrer un point relatif au personnage étudié et qu'il organise en conséquence son récit, son livre se situe aux frontières de l'histoire-problème.

La thèse de Droz est simple. Si les ouvriers français de cette époque se méfient des institutions politiques tout en combattant le gouvernement, c'est à cause de l'influence de Proudhon :

Cette transformation prodigieuse d'une mentalité nationale est la plus forte marque de l'ascendant de Proudhon et de son génie, un des effets les plus étonnants que jamais ait produits un homme. La recherche des causes en histoire est décevante. Cette fois, cependant, il semble qu'on ne peut attribuer ce changement d'un peuple, ou mieux d'une classe de ce peuple, représentée par son élite, que par les enseignements anarchistes de Proudhon. Et si les sentiments nouveaux, que Proudhon lui a comme imposés, subsistent, on pourra mesurer l'influence de l'instituteur à la vitalité de ce qu'il a su inspirer. ${ }^{64}$

Cette thèse, manifestement outrancière, fut critiquée par Lucien Febvre dans l'un de ses tout premiers textes. Dans un compte rendu, il reprochait à Droz d'établir des relations de cause à effet entre l'action des individus et le mouvement des institutions. Il s'agit là de deux ordres de réalité qui évoluent en étroite symbiose sans que l'on puisse attribuer à l'un la paternité de l'autre. ${ }^{65}$ Mais ce qu'il faut retenir du livre de Droz, ce n'est pas la thèse, c'est plutôt le fait que son récit est construit en fonction de cette thèse, qui repose elle-même sur une question implicite, mais quand même efficace, puisqu'elle préside à l'organisation de la matière historique : comment s'explique l'attitude politique du mouvement ouvrier français?

64. Droz, op, cit., 47-48.

65. Lucien Febvre, "Proudhon et le syndicalisme contemporain ", Revue de synthèse historique, XIX (1909) (179-193) reproduit dans Pour une histoire à part encière (Paris : SEVPEN, 1962.859 p.) (772-786) : 783-786. 
Léon Blum et les Congrès ouvriers et socialistes. Une question du même type sous-tend l'histoire des Congrès ouvriers et socialistes que nous présente Léon Blum. ${ }^{66}$ Dans cet ouvrage d'un militant à la recherche de ses origines, il n'y a pas de problème, ou d'hypothèse, explicitement formulé. Néanmoins le récit, tout affirmatif et positif qu'il soit, ne se dissimule pas derrière une absence d'interprétation. Il ne se prétend pas linéaire, sans relief et sans parti-pris, mais il est au contraire aménagé de façon à démontrer la vérité de la thèse suivante : que le socialisme collectiviste s'est progressivement imposé dans l'idéologie ouvrière sous l'effet de l'activité discursive des militants ouvriers eux-mêmes. Il utilise, pour effectuer cette démonstration, les débats qui eurent lieu à l'occasion des congrès ouvriers. Mais au lieu de résumer les débats d'une façon soi-disant impartiale, il les interprète en les situant dans leur contexte politique du moment et dans une perspective évolutive, historique.

Au premier congrès ouvrier de Paris en 1876, les organisateurs voulaient, affirme Blum, que « le Congrès gardât un caractère exclusivement ouvrier. Ils se méfiaient des politiciens. " "C'était là, dit-il, l'ancien esprit syndicaliste, et sa méfiance de l'action politique. Toutes les précautions furent donc prises pour que la représentation au Congrès fût purement ouvrière. ${ }^{67}$ Après avoir résumé et commenté les débats de ce Congrès, Blum en dégage le sens. "Il est manifeste, conclut-il, par tous ces faits, bien que peu cohérents, par ces formules encore vagues, que les congressistes de 1876 concevaient vraiment le parti ouvrier comme un parti de classe, son action comme une action de classe. Si ces quelques points n'étaient pas mis en lumière, l'bistoire des Congrès qui ont suivi celui de Paris deviendrait inintelligible. " ${ }^{68}$ L'ouvrage de Blum fourmille de ces remarques et de ces commentaires qui permettent de comprendre les Congrès ouvriers, qui leur restituent en réalité une logique et une unité. À propos du Congrès de 1878 : «Dans les discussions purement économiques, on sentait aussi le Congrès rapprocher peu à peu son

66. Léon Blum, Les Congrès ouvriers et socialistes français (Paris : Société nouvelle de librairie et d'édition, 1901. 4 vol.).

67. Blum, op. cit., 7 .

68. Blum, op. cit., 17. 
langage et sa pensée de la doctrine révolutionnaire [. . . ] On sentait chaque jour se dégager et s'éclaircir la notion du collectivisme. " 69

C'est le propre de l'histoire-problème de s'élever au-dessus du magma confus des mots et des choses pour en dégager un sens, une interprétation, qui sans être l'application intégrale d'une philosophie de l'histoire pensée sans référence particulière aux faits étudiés, porte en elle-même une conception du monde qui, appliquée à la compréhension d'un phénomène particulier, permet une compréhension significative de ce dernier. Blum ne résume pas platement les débats des Congrès ouvriers, il ne cherche pas non plus à montrer qu'ils sont le signe manifeste de la montée dans l'histoire de la classe ouvrière, il nous dit simplement, en nous le démontrant par des faits tirés de la réalité étudiée, qu'un fil conducteur, une logique interne se dégage de l'organisation et des travaux des Congrès: l'adhésion progressive des ouvriers français à la doctrine socialiste.

Une histoire des Bourses du travail. À l'instar de Léon Blum, Fernand Pelloutier, auteur d'une histoire des Bourses du travail, ${ }^{70}$ est un militant ${ }^{71}$ qui effectue un retour sur le passé, mais contrairement à Blum, qui réalisait une mise au point destinée sans doute à guider et à justifier son action future, Pelloutier livre ici une sorte de testament. Ayant été le principal animateur des Bourses du travail, il entend à la fin de sa vie - l'ouvrage est posthume - donner sa version de cette histoire. Comme le dit très bien Georges Sorel dans sa Préface : "Il est très facile de voir que Pelloutier n'a pas cherché à dissimuler un seul instant l'influence que sa conception personnelle du devenir a exercé sur ses jugements. " De toute façon, comme le dit aussi Sorel, "il est impossible d'échapper à cette nécessité du relativisme ". ${ }^{72}$ Les socialistes n'essaient pas de dissimuler cette contrainte, au contraire, ils l'exploitent à leur profit.

69. Blum, op. cit., 25.

70. Fernand Pelloutier, Histoire des Bourses du travail. Origine, Institutions, Avenir (Paris : Gordon \& Breach, 1971) (Paris : Costes, 1946) (1901). 340 p.).

71. A sa mort, Pelloutier était Secrétaire général de la Fédération des Bourses du Travail de France et des Colonies.

72. Pelloutier, op. cit., 29. 
Quand les socialistes parlent des institutions ouvrières, ils ne séparent jamais leurs appréciations de trois ordres de considérations: tendances qu'ils croient découvrir dans la société capitaliste, conditions dans lesquelles s'opérera d'après eux la rupture mettant fin au monde actuel, conjectures sur l'avenir. ${ }^{73}$

C'est donc un témoignage que nous apporte Pelloutier, un témoignage dont Sorel a lui-même spécifié les diverses finalités :

- faire profiter ses camarades de la grande expérience qu'il avait acquise dans sa pratique des organisations ouvrières

- montrer ce qu'elles peuvent quand elles sont bien pénétrées de la portée de leur véritable mission

- convaincre les travailleurs qu'ils trouveront facilement parmi eux les hommes capables de diriger leurs institutions, le jour où ils cesseront d'être hypnotisés par les utopies politiques

- apprendre au prolétariat à vouloir, l'instruire par l'action et lui révéler sa propre capacité. ${ }^{74}$

Ces considérations du préfacier sont importantes car elles explicitent très bien la nature profonde de cette manière d'écrire l'histoire qui interprète le passé en fonction du présent et de l'avenir, qui structure le récit en fonction de problèmes significatifs pour le présent. Telle est bien la caractéristique dominante de cette aeuvre dont la majeure partie est consacrée à décrire le fonctionnement des Bourses, mais qui comporte une longue première partie où elles sont situées par rapport à l'évolution historique du mouvement ouvrier. Celui-ci est analysé sous le double point de vue de sa constitution au sein de la société et du maintien de son autonomie par rapport au mouvement politique. $\grave{A}$ propos du premier Congrès ouvrier de 1876, qui marqua l'institutionalisation d'un mouvement syndical intégralement ouvrier, après l'hécatombe de la Commune, Pelloutier écrit :

N'était-ce pas que l'intuition populaire perçut dans l'association par classes l'unique moyen de transformation sociale et que, malgré ses sentiments de conciliation économique, malgré son apparente indifférence politique, sous l'empire, en quelque sorte, d'une clairvoyance irraisonnée, l'ouvrier devinât dans le

73. Pelloutier, op. cit., 29.

74. Pelloutier, op. cit., 27. 
communisme des idées et des intérêts à la fois l'instrument de destruction du despotisme et l'instrument d'édification de l'harmonie économique ? ${ }^{75}$

Naturellement, le mouvement syndical qui se développe assez rapidement par la suite fut sollicité par le socialisme, les politiques des partis ouvriers. Malheureusement, les militants politiques voulurent aller trop vite et se subordonner les syndicats :

En conséquence de cette faute, le fossé se creuse de plus en plus entre le Parti et les associations ouvrières. De temps à autre, quelque socialiste avisé préconisait l'entente; mais la faillite chaque jour plus évidente des sectes politiques et les dissentiments qu'introduisaient dans les syndicats les discussions sur l'action électorale détournaient ceux-ci d'un rapprochement dont ils pressentaient confusément qu'ils seraient les victimes. ${ }^{76}$

L'histoire de Pelloutier est donc agencée selon une problématique bien particulière. Elle ne prétend pas à l'exhaustivité, à l'impartialité. Au contraire, elle entend démontrer quelles sont les raisons, les motifs, les circonstances, qui ont amené les ouvriers français à se constituer en un mouvement syndical autonome, voué, à la limite, à la destruction du capitalisme et conservant par ailleurs son indépendance face au courant proprement politique du mouvement ouvrier. Ce n'est pas la philosophie marxiste de l'histoire que Pelloutier applique à la connaissance du mouvement ouvrier français, mais c'est une interprétation qui prend son sens dans la vie même de l'auteur et qui dépasse de loin la simple description des faits. Pelloutier, qui s'est battu toute sa vie pour le mouvement syndical dont l'avenir le préoccupe profondément, en a reconstitué l'histoire à partir de sa problématique de militant. Voilà une œuvre, qui sans avoir toute la rigueur de celle d'un historien de métier, porte en elle les principes même de la méthodologie de l'histoire-problème.

\section{HISTOIRE ET SCIENCES SOCIALES}

Le discours historique a connu depuis le $\mathrm{XIX}^{\text {ième }}$ siècle des mutations importantes au plan de son organisation

75. Pelloutier, op. cit., 73-74.

76. Pelloutier, op rit., 101-102 
formelle. L'histoire-problème et l'histoire structurale ont successivement contesté à l'histoire-récit la place quasi exclusive qu'elle détenait à la fin du siècle dernier au sein de la communauté des historiens. On a vu cependant qu'à l'intérieur de l'histoire-récit cohabitaient des manières différentes de composer le récit historique, liées à des philosophies de l'histoire particulières, et annonciatrices d'une autre forme de représentation historique, l'histoire-problème. Dès avant 1914, on voit également apparaître l'esquisse de ce qui deviendra l'histoire structurale, soit la forme que prendra l'histoire-problème quand elle aura davantage intégré le contenu et les méthodes des sciences sociales, dont elle s'inspire.

À la fin du XIX ${ }^{\text {ième }}$ siècle, le positivisme alors florissant offre deux possibilités à l'historien : ou bien prétendre que l'histoire ne pourra jamais être une science sinon par la reconstitution érudite des faits; ou bien vouloir analyser le passé avec la même rigueur et des schèmes de même nature que ceux des sciences naturelles, ou tout au moins des sciences sociales naissantes. Dans les deux cas, l'histoire était condamnée, soit à disparaître de par l'inanité de ses prétentions, soit à être dévorée par les nouvelles sciences de l'homme, notamment par la sociologie.

Dans les deux cas, ce positivisme réagissait contre la philosophie de l'histoire et si la réaction anti-positiviste atténua cet anathème, ${ }^{77}$ ce qui permit à l'histoire-problème de s'instituer et de dépasser l'érudition sans être à la remorque des sciences sociales, le problème des rapports de l'histoire avec ces dernières reste entier. ${ }^{78}$ L'historien doit étudier le passé à la lumière des questions que soulève, que pose le présent, mais ne peut-il pas utiliser les grilles, les méthodes, les outils conceptuels des sciences sociales? Il s'agit en réalité d'un problème identique à celui du rapport entre les visions du

77. H. Stuart Hughes, Consciousness and Society. The Reorientation of European Social Thought 1890-1930 (Toronto : Random House, 1958. xi-433-xv p.).

78. Voir le livre de Paul Veyne, et le débat qu'il a suscité, Le pain et le cirque. Sociologie bistorique d'un pluralisme politique (Paris : Seuil, 1976. 799 p.); Jean Andreau, Pauline Schmitt et Alain Schnapp, "Paul Veyne et l'évergétisme ", Annales E.S.C., XXXIII (1978) (307-325); François Hartog; "Paul Veyne naturaliste: l'histoire est un herbier ", Annales E.S.C., XXXIII (1978) (326-330). 
monde propres à la philosophie de l'histoire et la connaissance des faits concrets, sauf qu'il faut maintenant articuler les visions du monde propres aux sciences sociales avec la connaissance des faits concrets du passé.

Un bel exemple de cette problématique épistémologique à son état d'émergence nous est donné avec l'ouvrage de Maxime Leroy, consacré à La coutume ouvrière. ${ }^{79}$ Leroy tient la science en haute estime. Si Levasseur croyait à la science comme facteur de productivité économique, il pense pour sa part que les conflits sociaux pourraient se résorber si les classes sociales avaient une meilleure connaissance de leurs positions respectives :

Les classes s'ignorent réciproquement parce que chacune agit dans la dépendance de sa situation économique et politique. Diverses, antagonistes, elles pensent diversement, antagoniquement. Séparées les unes des autres par des croyances et des intérêts, elles marquent, d'un camp à l'autre, ces intérêts et ces croyances du signe de mauvaise foi et les appellent des préjugés : si elles se connaissaient, elles s'apprécieraient, et elles seraient presque confondues; si elles discutaient, elles seraient bien près de s'entendre. ${ }^{80}$

Leroy reconnaît que la pensée sociale s'articule aux structures économiques, mais il conserve un préjugé favorable envers la science et la raison, capables jusqu'à un certain point de réconcilier des positions antagonistes et de suggérer des voies d'issue. Il ne va pas, cependant, à l'instar de Levasseur ou des marxistes les plus orthodoxes, jusqu'à postuler l'existence d'une vérité objective. D'une part, il exprime très clairement sa position personnelle : "nous dirons donc que nous avons écrit ce livre avec sympathie pour le régime d'égalité et de liberté, basé sur le travail, que les syndicats préparent patiemment depuis la scission qui s'est produite entre producteurs et bénéficiaires de la production $"{ }^{81}$ D'autre part, il énonce un point de vue général sur cette question de l'objectivité. "Un auteur, un savant rapportant ses expériences, un observateur, un historien, un sociologue ne peuvent à vrai dire échapper à leurs préférences de philosophe

79. Maxime Leroy, La coutume ouvrière (Paris : Giard et Brière, 1913. 2 vol.).

80. Leroy, op. cit., 5.

81. Leroy, op. cit., 7 . 
ou de citoyen. On ne doit exiger d'eux, à ce point de vue, que d'avoir voulu être sincères, documentés avec probité. ${ }^{82} \mathrm{Et}$ encore, n'est-ce pas là non plus une condition particulière aux sciences humaines. "Le chimiste croit ne voir que des corps en fusion ; en réalité, une hypothèse bouillonne devant ses yeux ; particulière, elle se relie à une hypothèse plus étendue, enfin à un système cosmogonique qui, par sa généralité, est moins une explication technique qu'une véritable philosophie, incertaine et tendancieuse. ${ }^{83}$

C'est là une position bien proche de celle de Lucien Febvre, qui essaie de réconcilier l'adhésion à une vision du monde avec la poursuite de l'objectivité, et par-delà, avec la scientificité, en démystifiant le caractère absolu du postulat d'objectivité, sur lequel reposait l'épistémologie scientifique positiviste. ${ }^{84}$ Certes, cette position est doublement ambiguë, dans la mesure où elle implique une certaine distanciation par rapport à certaines conceptions particulières de l'histoire qui s'approprient la vérité, qu'elles soient libérale ou marxiste, et aussi une démarcation par rapport à une conception proprement idéalisée de la scientificité - ou alors, dans le cas de l'histoire, exclusivement érudite. Mais c'est la seule attitude possible si l'on veut pratiquer une science utile, enracinée dans le présent, et ouverte sur le futur, qui ne postule pas un univers clos et entièrement déterminé.

Cette difficulté n'est pas liée à la discipline historique, même si l'historien qui s'identifie à des pratiques et à des savoirs la vit avec intensité. Dans l'esprit de Leroy, elle est propre à toute connaissance qui interfere de près ou de loin avec une conception du monde. Néanmoins, Leroy pousse plus loin sa réflexion épistémologique et nous amène cette fois sur la frontière entre histoire et sciences sociales. "Écrit par un juriste, dit-il, ce livre n'est pas une histoire du mouvement ouvrier: il ne faut donc pas y chercher les renseignements complets sur l'évolution syndicale. L'histoire de cette évolution a été faite déjà, plusieurs fois, à un point de vue général et interprofessionnel [. . . ] ${ }^{85}$ Cette observation est fort signifi-

82. Leroy, op. cit., 7. 8.

83. Leroy, op. cit. 7.8.

84. Lucien Febvre, Combats pour l'bistoire (Paris : Colin, 1965 (1953). ix-458 p.).

85. Leroy, op. cit., 9. 
cative, car elle identifie l'histoire à l'information et à l'exhaustivité. Plutôt qu'un travail historique, il a "voulu tenter l'œuvre nouvelle d'une systématisation de la pratique syndicale actuelle ". "Toutefois, poừrsuit-il, chaque chapitre de ce répertoire du droit prolétarien'est précédé d'une note historique dont l'objet est de situer dans le temps les règles et les institutions ouvrières. " ${ }^{86}$ C'est ainsi que l'histoire devient, de par sa méthode et son matériau, un complément, un renforcement à l'approche sociologique. On le voit plus nettement encore dans le passage suivant :

Il y a quelques années, au temps où les sociologues cherchaient leurs principes, leur méthode et leurs comparaisons dans les sciences naturelles, nous aurions écrit, pour bien faire comprendre ce dessein, que notre livre était un essai tout à la fois d'embryogénie et de morphologie sociales. Aujourd'hui, loin des anciennes disciplines, nous dirons simplement que nous avons tenté une explication descriptive de la coutume ouvrière, selon la méthode générale proposée par la nouvelle sociologie qui s'est mise à l'école de l'histoire. ${ }^{87}$

Dans cet ouvrage de Leroy, le problème des rapports entre histoire et sciences sociales est posé du point de vue de la sociologie. Mais quel que soit le point de vue, le problème est posé et restera au cour de l'épistémologie de la discipline historique jusqu'à nos jours. Jusqu'à quel point l'histoire peut-elle se faire «systématisation», pour reprendre l'expression de Leroy, sans trahir son objet ou se trahir elle-même ? Ce problème très actuel s'esquissait déjà au début du siècle dans l'historiographie ouvrière.

Cette situation mitoyenne d'une sociologie qui s'est mise à l'école de l'histoire, qui fait appel au complément historique permettant de situer les réalités dans leur contexte temporel, se reflète dans la définition de l'objet mis en cause dans cette œuvre. Leroy indique au début de son étude l'analogie qui existe entre le droit bourgeois, c'est-à-dire les règles inventées par la bourgeoisie en marge du droit d'ancien régime, et l'organisation ouvrière et sa doctrine qui se sont développées en marge du droit civil, les deux mouvements étant le fait, à des époques différentes, « de la classe la plus proche du travail ».

86. Leroy, op. cit., 10

87. Leroy, op. cit., 10. 
Pour l'heure, «cette doctrine est connue, c'est le communisme, le collectivisme; dans sa dernière forme, le syndicalisme. On a étudié cette doctrine, la valeur des Syndicats, leur force de résistance, leur nombre ; on a enregistré leurs défaites, leurs victoires. Ce qui a été laissé de côté, c'est tout ce qui concerne leur vie intérieure, leur vie juridique autonome. ${ }^{88}$

Le plus remarquable est que Leroy invoque à l'appui de sa constatation l'étude de Philippe Sagnac sur La législation civile de la Révolution française, ${ }^{89}$ ainsi que l'œuvre de Tocqueville, L'Ancien Régime et la Révolution. ${ }^{90}$ Ce sont là deux historiens qui ont étudié pour d'autres temps la même réalité que Leroy. Or, Sagnac, dans ce travail particulier, et Tocqueville peuvent être considérés, à l'exemple de Leroy, comme des historiens sociologues, ou des sociologues historiens. Comme quoi, à moins de reléguer l'histoire dans l'érudition pure et simple, l'histoire et les sciences sociales se côtoient et se mêlent aisément. Ce qui ne doit pas nous faire oublier le problème de fond : la systématisation, la conceptualisation qu'implique la jonction avec les sciences sociales sont-elles compatibles avec la mise en valeur de l'historicité ? Autrement dit: l'histoire a-t-elle un lieu et une fonction propres entre la description exhaustive de la réalité et l'imitation des sciences humaines?

\section{CONCLUSION}

L'organisation du discours et la représentation du contenu de la matière historique, s'articulent et constituent des types de pratique historique que nous avons analysés à travers les premiers écrits de l'historiographie ouvrière française. La typologie générale, histoire-récit, histoire-problème, histoire structurale, se retrouve, à l'état embryonnaire tout au moins, dès cette époque, et nous permet de mettre en relief la position irrémédiablement équivoque du discours historique. Entre le présent et le passé, il risque de n'être qu'un catalogue informatif, faussement impartial, ou une sociologie qui s'en

\footnotetext{
88. Leroy, op. cit., 26.

89. Philippe Sagnac, La législation civile de la Révolution française (1789-1904). Essai d'bistoire sociale (Paris: Hachette, 1898. xx-446 p.).

90. Alexis De Torqueville, LAncien Régime et la Révolution (Paris: Gallimard, 1967. 378 p.).
} 
refuse le nom, ou alors il assume pleinement son rôle de réécrire sans cesse le passé en fonction du présent, mais dans ce cas, il lui faut éviter de n'être que le complément d'une philosophie déterministe de l'histoire qui enlève à l'historicité toute raison d'être. Il ne lui reste, en définitive, que l'histoire ouverte, indéterminée dans son organisation et son déroulement, mais non dénuée ni de sens, ni de logique, appelant sans cesse à de nouveaux questionnements sur les causes et les significations. Seule l'histoire-problème, et ce qui s'en approche, permet d'assumer cette position difficile, qui est celle-là même de la liberté.

Université du Québec à Rimouski 